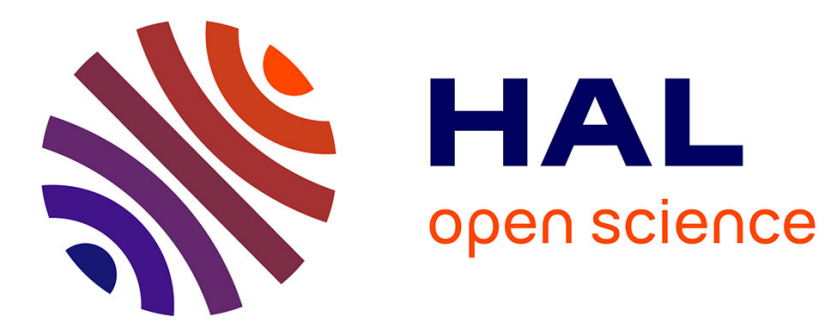

\title{
Dynamics of a structured neuron population
}

Khashayar Pakdaman, Benoît Perthame, Delphine Salort

\section{To cite this version:}

Khashayar Pakdaman, Benoît Perthame, Delphine Salort. Dynamics of a structured neuron population. 2009. hal-00387413

\section{HAL Id: hal-00387413 https://hal.science/hal-00387413}

Preprint submitted on 25 May 2009

HAL is a multi-disciplinary open access archive for the deposit and dissemination of scientific research documents, whether they are published or not. The documents may come from teaching and research institutions in France or abroad, or from public or private research centers.
L'archive ouverte pluridisciplinaire HAL, est destinée au dépôt et à la diffusion de documents scientifiques de niveau recherche, publiés ou non, émanant des établissements d'enseignement et de recherche français ou étrangers, des laboratoires publics ou privés. 


\title{
Dynamics of a structured neuron population
}

\author{
Khashayar Pakdaman * Benoît Perthame ${ }^{\dagger \ddagger} \quad$ Delphine Salort *§
}

February 18, 2009

\begin{abstract}
We study the dynamics of assemblies of interacting neurons. For large fully connected networks, the dynamics of the system can be described by a partial differential equation reminiscent of agestructure models used in mathematical ecology, where the "age" of a neuron represents the time elapsed since its last discharge. The nonlinearity arises from the connectivity $J$ of the network.

We prove some mathematical properties of the model that are directly related to qualitative properties. On the one hand we prove that it is well-posed and that it admits stationary states which, depending upon the connectivity, can be unique or not. On the other hand, we study the long time behavior of solutions; both for small and large $J$, we prove the relaxation to the steady state describing asynchronous firing of the neurons. In the middle range, numerical experiments show that periodic solutions appear expressing re-synchronization of the network and asynchronous firing.
\end{abstract}

Key-words Structured population dynamics, neurons populations, nonlinear asymptotic stability, synchronization.

AMS Class. No. 35F15, 35B10, 92B20.

\section{Introduction}

Synchronous regular or irregular activities are ubiquitous in nervous systems and can be at the basis of physiological functions such as respiration, cognitive function or pathological conditions such as epilepsy. These synchronous patterns reflect simultaneous and repetitive discharges of large numbers of neurons in specific assemblies at the level of the brainstem, the cortex, etc. Such coherence results from the interplay between the intrinsic neural properties and the coupling principles underlying the network. A number of theoretical investigations have aimed to determine the mechanisms underlying the genesis of synchronous firing in neural assemblies, through both numerical and analytical studies.

One mechanism for obtaining global coherent activity in assemblies is through the interconnection of self oscillating units. Synchrony in such systems strongly depends on the distribution of the frequencies of spontaneous oscillations of the components. This form of oscillation has been the topic of numerous investigations. However, it is also possible to obtain coherent global activities in assemblies that do

\footnotetext{
*Institut J. Monod email: pakdaman@ijm.jussieu.fr

${ }^{\dagger}$ UPMC Univ Paris 06, UMR 7598, Laboratoire Jacques-Louis Lions, F-75005, Paris, France and Institut Universitaire de France. Email: benoit.perthame@upmc.fr

${ }^{\ddagger}$ CNRS, UMR 7598, Laboratoire Jacques-Louis Lions, F-75005, Paris, France

$\S$ email: salort.delphine@ijm.jussieu.fr
} 
not contain such pacemaker units. neural assemblies constitute one example where such dynamics have been reported. This paper is mainly concerned with this issue.

Early, experimental motivation for this paper came from recordings that monitored the spontaneous activity of brainstem slices. Simulation of a computational model adjusted to the experiments revealed that the network could sustain regular rhythmic activity in some parameter ranges. Similar observations had been made in [14] for models of long lasting transients observed in the cerebral cortex. Since the earlier studies in $[12,13]$, several other studies obtained such results. That the phenomenon can be observed in many models suggests that the fine details of the model may not be at the core of the mechanism, and that to get the gist of the phenomena, one may focus on a few features of neural dynamics. Indeed, the systematic exploration of the influence of model parameters hints that the key ingredient is the relation between the recovery time scale of the neuron and the input time scales. Furthermore, the numerical simulations show that the phenomenon persists in networks with diverse connectivity. For instance while the first studies in $[12,13]$ where performed in randomly connected networks, the same phenomenon can also be observed in fully connected homogenous networks. These simulations show several points, first that the effect observed in finite size stochastic models appears in the form of oscillations in the averaged model obtained in the limit of infinite sized population. Second that in this version, to the different regimes correspond different asymptotic regimes of the system. In summary, these numerical simulations point to the mathematical analyses of the population models as one way of gaining insight into the mechanisms that control the genesis of various forms of activity in neural populations.

This paper is concerned with such mathematical analyses of a continuous model for a random neural assembly. We first introduce the model and show that it exhibits self-sustained oscillations in a certain range of a parameter $J$ measuring the network connectivity. For mathematical analysis, we consider a particularly simple form of interactions, that preserves the main property of the model regarding self-sustained oscillations and for which we can prove existence of solutions. We show that it exhibits relaxation to a steady state when $J$ is small or $J$ is large. Since a population of individual neuron $(J=0)$ has the property of desynchronization, the interpretation for $J$ small is clear. For $J$ large it means that too many connections also restore this desynchronization of the assembly.

After a presentation of the model in section 2, we analyze the steady states (section 3) and recall a method to prove the resynchronization property (with exponential rate) for disconnected networks (section 4). Existence of solutions to the nonlinear system for the assemblies of neurons is proved afterwards in section 5 and this brings material to show global nonlinear asymptotic stability both for small and large connectivity, this is performed in section 6 . We conclude with numerical simulations that illustrate our theoretical results and the existence of self-sustained oscillations in the middle range of the connectivity parameter.

\section{The neuron population model}

We consider a population of neurons described by the probability density $n(s, t)$ of finding a neuron in 'state' $s$ at time $t$ where $s$ represents the time elapsed since the last discharge. According to [12, 13] 
a simple model of the dynamics is given by the age-structured equation

$$
\left\{\begin{array}{l}
\frac{\partial n(s, t)}{\partial t}+\frac{\partial n(s, t)}{\partial s}+p(s, X(t)) n(s, t)=0, \\
N(t):=n(s=0, t)=\int_{0}^{+\infty} p(s, X(t)) n(s, t) d s
\end{array}\right.
$$

completed with an initial probability density $n^{0}(s)$ that satisfies

$$
0 \leq n^{0}(s) \leq 1, \quad \int_{0}^{\infty} n^{0}(s) d s=1
$$

The coefficient $p(s, X)$ represents the firing rate of neurons in the 'state $s$ ' and in an environment $X$ resulting from the global neural activity. It is usually small (or even vanishes) during a rest phase that depends on $X$, and increases suddenly afterwards. The density of neurons undergoing a discharge at time $t$ is denoted by $N(t)$ and the boundary condition at $s=0$ means that the neuron re-enters the cycle at 'age $s=0$ ' after firing.

The interactions between the neurons are taken into account through the global neural activity at time $t, X(t)$, that is given by

$$
X(t)=J \int_{0}^{t} \alpha(u) N(t-u) d u
$$

The parameter $J \geq 0$ represents the network connectivity (and thus the strength of the interactions), and the delay takes into account an averaged propagation time for the ionic pulse in this network. Here we assume that the neurons can discharge earlier when the neural activity is high.

Notice that this nonlinear system has a remarkable conservation property

$$
\int_{0}^{\infty} n(s, t) d s=\int_{0}^{\infty} n^{0}(s) d s=1 \quad \forall t \geq 0
$$

which expresses the interpretation of $n(t, \cdot)$ as a probability density.

These modeling assumptions can be written on the (nonnegative) coefficients as

$$
\begin{gathered}
\int_{0}^{\infty} \alpha(u) d u=1, \quad \alpha(\cdot) \geq 0, \\
\frac{\partial}{\partial s} p(s, x) \geq 0, \quad p(s, x)=0 \text { for } s \in\left(0, s^{*}(x)\right) \quad p(s, x)>0 \text { for } s>s^{*}(x), \quad p(s, x) \stackrel{s \rightarrow \infty}{\longrightarrow} 1, \\
\frac{\partial}{\partial x} p(s, x) \geq 0, \quad p(s, x) \underset{x \rightarrow \infty}{\longrightarrow} 1 .
\end{gathered}
$$

In order to simplify these assumptions and the mathematical analysis, throughout the paper we often use the particular firing rate

$$
\left\{\begin{array}{l}
p(s, x)=\mathbf{1}_{\left\{s>s^{*}(x)\right\}}, \quad \text { for some } s^{*} \in C^{1}, \quad \frac{d}{d x} s^{*}(x) \leq 0, \\
s^{*}(0)=s_{+}^{*}, \quad s^{*}(\infty)=s_{-}^{*}<s_{+}^{*}<1 .
\end{array}\right.
$$

A discontinuity in this coefficient is not realistic but does not change the behavior of solutions that we describe below; it has the advantage of allowing for simpler explicit expressions and thus it simplifies 
the proofs. In the range $x \approx 0$, the value $s_{+}^{*}$ represents the spontaneous activity due to noise. In the range $x \gg 1$ we will use the decay rates $D$ and $\widetilde{D}$ defined by

$$
\begin{gathered}
\limsup _{J \rightarrow \infty} \sup _{x \geq \frac{J}{\left(1+s_{+}^{*}\right)}} \frac{J\left|s^{* \prime}(x)\right|}{\left(1+s^{*}(x)\right)^{2}}:=D, \\
\quad \limsup _{J \rightarrow \infty} \sup _{x \in \mathcal{C}_{J}} J\left|s^{* \prime}(x)\right|:=\widetilde{D}
\end{gathered}
$$

where

$$
\mathcal{C}(J)=\left[J\left(1-s_{+}^{*}\right), J\right]
$$

Later on, we will use the condition that the constants $D$ and $\widetilde{D}$ are small enough, this means that $s^{*}(x) \rightarrow s_{-}^{*}$ fast enough as $x \rightarrow \infty$. This holds for example if $s^{*}(x)=s_{-}^{*}$ for $x$ large enough or more generally if $\left|s^{* \prime}(x)\right| \leq 1 / x^{2}$ (then $D=0$ and $\widetilde{D}=0$ ). The condition $D<1$ or $\widetilde{D}<1$ corresponds to $\left|s^{* \prime}(x)\right| \leq A / x$ with $A$ small enough.

The equation (1) is an original nonlinear version of a standard linear equation, the renewal equation. It arises in various biological applications as epidemic spread, cell division cycle or even ecology (see $[6,11,2])$.

Our goal is to study the existence of solutions to (1) and their long time behavior. Before we consider the steady states and the linear equation corresponding to $X \equiv 0$.

\section{Steady states}

Let $J \geq 0$ and assume the simple situation (8). A function $A_{J}(s)$ associated with the neural activity $X_{J}^{*}$, is a unit mass stationary solution to (1), (3) if and only if

$$
\left\{\begin{array}{l}
\frac{\partial A_{J}}{\partial s}+p\left(s, X_{J}^{*}\right) A_{J}=0 \\
A_{J}(0)=\left(1+s^{*}\left(X_{J}^{*}\right)\right)^{-1} \\
X_{J}^{*}=J\left(1+s^{*}\left(X_{J}^{*}\right)\right)^{-1}
\end{array}\right.
$$

Indeed we have

$$
A_{J}(s)=A_{J}(0) e^{-\int_{0}^{s} p\left(\sigma, X_{J}^{*}\right) d \sigma}, \quad A_{J}(0)=\left(\int_{0}^{\infty} e^{-\int_{0}^{s} p\left(\sigma, X_{J}^{*}\right) d \sigma} d s\right)^{-1}=\left(1+s^{*}\left(X_{J}^{*}\right)\right)^{-1} .
$$

Consequently, the third equation of (12) defines steady states and, from our assumptions on $s^{*}(x)$, for all $J$ there is at least one steady state. It is easy to see that there can be several steady states when $s^{*}(x)$ has large enough derivative.

Uniqueness is equivalent to find a unique $X_{J}^{*}$ such that

$$
X_{J}^{*}=J\left(1+s^{*}\left(X_{J}^{*}\right)\right)^{-1}
$$

Therefore uniqueness always holds true for $J$ small enough. Uniqueness of the steady state also holds true, for all $J \geq 0$, under the condition

$$
\frac{J\left|s^{* \prime}(x)\right|}{\left(1+s^{*}(x)\right)^{2}}<1, \quad \forall x>0
$$

but such a regime is very restricitive. We can relax it and notice that 
Lemma 3.1 With the assumptions (8), the steady state is unique for $J$ large enough under the condition $D<1$ in (9).

Proof of Lemma 3.1. Indeed, using (8), a steady state satisfies $X_{J}^{*} \geq J\left(1+s_{+}^{*}\right)^{-1}$. If there are two steady states at least, then there is a point $Y$ in between such that

$$
1=-\frac{J s^{* \prime}(Y)}{\left(1+s^{*}(Y)\right)^{2}}
$$

which means $J \geq J_{0}$, the largest value of $J$ such that $\sup _{x \geq J /\left(1+s_{+}^{*}\right)} \frac{J s^{* \prime}(x)}{\left(1+s^{*}(x)\right)^{2}} \geq 1$ which exists thanks to our assumption on $D$.

\section{Asymptotic stability of the steady states for $J=0$}

For $J=0$ the system is linear and reads, with the short hand notation $\bar{p}(s)=p(s, 0)$,

$$
\left\{\begin{array}{l}
\frac{\partial n(s, t)}{\partial t}+\frac{\partial n(s, t)}{\partial s}+\bar{p}(s) n(s, t)=0, \\
N(t):=n(s=0, t)=\int_{0}^{\infty} \bar{p}(s) n(s, t) d s, \\
n(s, 0)=n^{0}(s) \geq 0, \quad \int_{0}^{\infty} n^{0}(s) d s=1 .
\end{array}\right.
$$

This linear equation admits a unique one parameter multiplicative family of steady states, we normalize to achieve unit mass

$$
A(s)=A(0) e^{-\int_{0}^{s} \bar{p}}, \quad A(0)=\left(\int_{0}^{\infty} e^{-\int_{0}^{s} \bar{p}} d s\right)^{-1} .
$$

A first step is to show that this state is exponentially stable, i.e., that for long times all solutions to (13), whatever the initial data, converge exponentially to $A(s)$ (thanks to the conservation property (4)). This property is known to follow from Laplace transform methods [5] which are not easy to extend to nonlinear situations and which do not provide a norm to establish the convergence. Another approach is through delay equations. For the case of neural models, see [7] and for a general approach see [3]. We rather use the entropy method introduced in [9]. The difficulty is that we need some kind of spectral gap property to arrive to an exponential convergence rate, which leaves some space for treating the nonlinear case later.

Theorem $4.1(J=0)$ Assume $\bar{p}(s)=\mathbf{1}_{\left\{s>s^{*}\right\}}$ with $s^{*}<1$, then there is a $\mu>0$ and a function $\psi(s) \geq B>0$ such that

$$
\int_{0}^{\infty} \psi(s)|n(s, t)-A(s)| d s \leq e^{-\mu t} \int_{0}^{\infty} \psi(s)\left|n^{0}(s)-A(s)\right| d s .
$$

Proof of Theorem 4.1. We set $\widetilde{n}(s, t)=n(s, t)-A(s)$. It still satisfies the equation (13) by linearity. Therefore, we also have

$$
\frac{\partial|\widetilde{n}(s, t)|}{\partial t}+\frac{\partial|\widetilde{n}(s, t)|}{\partial s}+\bar{p}(s)|\widetilde{n}(s, t)|=0 .
$$


Let $\nu \in(0,1), \mu>0$ and $\psi(s)>0$ satisfy (we still have to show this is possible and this is the lemma 4.2 below)

$$
\frac{\partial \psi}{\partial s}-(\bar{p}(s)-\mu) \psi=-\psi(0)|\bar{p}(s)-\nu|
$$

Then we have (recall the conservation (4) which here tells us that $\int_{0}^{\infty} \widetilde{n}(s, t) d s=0$ )

$$
\begin{gathered}
\frac{d}{d t} \int_{0}^{\infty} \psi(s)|\widetilde{n}(s, t)| d s+\int_{0}^{\infty}\left[-\frac{\partial \psi(s)}{\partial s}+\bar{p}(s) \psi(s)\right]|\widetilde{n}(s, t)| d s= \\
=\psi(0)\left|\int_{0}^{\infty} \bar{p}(s) \widetilde{n}(s, t)\right| \\
=\psi(0)\left|\int_{0}^{\infty}[\bar{p}(s)-\nu] \widetilde{n}(s, t)\right| \\
\leq \psi(0) \int_{0}^{\infty}|\bar{p}(s)-\nu||\widetilde{n}(s, t)| .
\end{gathered}
$$

Then, the supposed equation on $\psi$ gives

$$
\frac{d}{d t} \int_{0}^{\infty} \psi(s)|\widetilde{n}(s, t)| d s=-\mu \int_{0}^{\infty} \psi(s)|\widetilde{n}(s, t)| d s
$$

and the result follows.

Lemma 4.2 Let $\bar{p}(s)$ given by $\bar{p}(s)=\mathbf{1}_{\left\{s>s^{*}\right\}}$ with $s^{*}<1$ and $0<\nu \leq 1$. Then, there exists $\mu>0$ such that equation (14) has a uniformly positive solution $\psi$ normalized with $\psi(0)=1$. Moreover, for the range $0<s_{-}^{*} \leq s^{*} \leq s_{+}^{*}<1$, there is a uniform constant $B>0$ such that $\psi(s) \geq B$.

Proof of Lemma 4.2. Let $0<\mu<1$ and choose $\psi(0)=1$.

On $\left(0, s^{*}\right)$, we have $\psi^{\prime}(s)=-\mu \psi-\nu$, i.e.,

$$
\psi(s)=e^{-\mu s}\left(1+\frac{\nu}{\mu}\right)-\frac{\nu}{\mu} .
$$

On $\left(s^{*}, \infty\right)$, we have $\psi^{\prime}(s)=(1-\mu) \psi-(1-\nu)$, and this gives exponential growth as soon as

$$
(1-\mu) \psi\left(s^{*}\right)=(1-\mu)\left(e^{-\mu s^{*}}\left(1+\frac{\nu}{\mu}\right)-\frac{\nu}{\mu}\right)>1-\nu .
$$

Thanks to Taylor expansion in $\mu$ at 0 , we obtain that

$$
(1-\mu)\left(1-\nu s^{*}+\varepsilon(\mu)\right)>1-\nu
$$

with $\lim _{\mu \rightarrow 0} \varepsilon(\mu)=0$. Hence it is enough to check that

$$
(1-\mu)\left(1-\nu s^{*}\right)>1-\nu .
$$

This holds true for $\mu$ small enough, because $s^{*}<1$. Lemma 4.2 is proved. 


\section{$5 \quad$ Existence and a priori estimates}

Before we study its stability, we first establish a general existence theorem for the nonlinear problem under consideration.

Theorem 5.1 Assume (2). Assume furthermore that there exists $q>1$ such that $\alpha \in L^{q}\left(\mathbb{R}^{+}\right)$. Then, there exists a unique solution to (1), (3), $n \in C\left(\mathbb{R}^{+} ; L^{1}\left(\mathbb{R}^{+}\right)\right)$and

$$
\begin{gathered}
0 \leq n(s, t) \leq 1, \quad \forall t, s \geq 0 \\
0 \leq N(t) \leq 1, \quad X(t) \in \mathcal{C}(J), \quad \forall t \geq 0
\end{gathered}
$$

where $\mathcal{C}(J)$ is defined by (11).

The assumption $\alpha \in L^{q}\left(\mathbb{R}^{+}\right)$with $q>1$ is crucial in our proof. However the case with no delay and more generally fixed delay, $\alpha(s)=\delta(s=\tau)$, can be handled by the same ideas when the firing rate $p$ is Lipschitz continuous in $s$.

Proof of Theorem 5.1. To prove wellposedness of a solution to (1), we are going to use a fixed point argument.

More precisely, we prove that there is a $T>0$ such that the following application

$$
\left\{\begin{array}{l}
F: L^{\infty}([0, T]) \rightarrow L^{\infty}([0, T]) \\
F(Y(\cdot))(t):=J \int_{0}^{t} \alpha(u) N(t-u) d u
\end{array}\right.
$$

admits a fixed point, where $N$ is given by

$$
N(t):=n(s=0, t)=\int_{0}^{+\infty} p(s, Y(t)) n(s, t) d s
$$

and where $n$ is the solution of the equation

$$
\left\{\begin{array}{l}
\frac{\partial n(s, t)}{\partial t}+\frac{\partial n(s, t)}{\partial s}+p(s, Y(t)) n(s, t)=0 \\
N(t):=n(s=0, t)=\int_{0}^{+\infty} p(s, Y(t)) n(s, t) d s
\end{array}\right.
$$

completed with the initial data $n^{0}$ satisfying (2). A fixed point of $F$, based on the Banach-Picard fixed theorem gives a unique solution on $[0, T]$ to the equation (1). Iterating on $T$ (which does not depend upon the initial data as we see it below), we deduce that in fact we have global existence and uniqueness of solutions of equation (1).

We are reduced to proving the following Lemma.

Lemma 5.2 Let $J \geq 0$. Then, there exists $T>0$ such that for all $\left(X_{1}, X_{2}\right) \in L^{\infty}([0, T])$, the following estimate holds

$$
\left\|F\left(X_{1}\right)-F\left(X_{2}\right)\right\|_{L^{\infty}([0, T])} \leq \frac{1}{2}\left\|X_{1}-X_{2}\right\|_{L^{\infty}([0, T])} .
$$


Proof of Lemma 5.2. We use the characteristics. For all $s \geq 0$, the function $\widetilde{n}_{s}(t):=n(s+t, t)$ is a solution of the equation

$$
\frac{d}{d t} \widetilde{n}_{s}(t)=-p(t+s, Y(t)) \widetilde{n}_{s}(t)
$$

We deduce that

$$
\widetilde{n}_{s}(t)=n^{0}(s) e^{-\int_{0}^{t} p\left(t^{\prime}+s, Y\left(t^{\prime}\right)\right) d t^{\prime}}
$$

and thus

$$
n(s, t)=n^{0}(s-t) e^{-\int_{0}^{t} p\left(t^{\prime}+s-t, Y\left(t^{\prime}\right)\right) d t^{\prime}} \quad \text { for } s \geq t .
$$

On the other hand, for all $t \geq 0$ we call $\widetilde{n}_{t}(s):=n(s, s+t)$. Then,

$$
\frac{d}{d s} \widetilde{n}_{t}(s)=-p(s, Y(t+s)) \widetilde{n}_{t}(s) .
$$

We deduce that

$$
n(s, s+t)=N(t) e^{-\int_{0}^{s} p\left(s^{\prime}, Y\left(s^{\prime}+t\right)\right) d s^{\prime}},
$$

and, as before, we deduce that

$$
n(s, t)=N(t-s) e^{-\int_{0}^{s} p\left(s^{\prime}, Y\left(s^{\prime}+t-s\right)\right) d s^{\prime}} \quad \text { for } s \leq t .
$$

We denote by $n_{i}, i=1,2$ two solutions of the equation

$$
\left\{\begin{array}{l}
\frac{\partial n_{i}(s, t)}{\partial t}+\frac{\partial n_{i}(s, t)}{\partial s}+p\left(s, X_{i}(t)\right) n_{i}(s, t)=0, \\
N_{i}(t):=n_{i}(s=0, t)=\int_{0}^{+\infty} p\left(s, X_{i}(t)\right) n_{i}(s, t) d s,
\end{array}\right.
$$

still with the initial data $n^{0}$. From (19) and (20), we deduce that

$N_{i}(t)=\int_{0}^{t} p\left(s, X_{i}(t)\right) N_{i}(t-s) e^{-\int_{0}^{s} p\left(s^{\prime}, X_{i}\left(s^{\prime}+t-s\right)\right) d s^{\prime}} d s+\int_{t}^{+\infty} n^{0}(s-t) p\left(s, X_{i}(t)\right) e^{-\int_{0}^{t} p\left(t^{\prime}+s-t, X_{i}\left(t^{\prime}\right)\right) d t^{\prime}} d s$.

We deduce that

$$
F\left(X_{1}\right)(t)-F\left(X_{2}\right)(t)=J \int_{0}^{t} \alpha(u)(H+E)(t-u) d u
$$

where

$$
\begin{aligned}
H(t)= & \int_{0}^{t} N_{1}(t-s) p\left(s, X_{1}(t)\right) e^{-\int_{0}^{s} p\left(s^{\prime}, X_{1}\left(s^{\prime}+t-s\right)\right) d s^{\prime}} d s \\
& -\int_{0}^{t} N_{2}(t-s) p\left(s, X_{2}(t)\right) e^{-\int_{0}^{s} p\left(s^{\prime}, X_{2}\left(s^{\prime}+t-s\right)\right) d s^{\prime}} d s,
\end{aligned}
$$

and $E(t)$ denotes the remaining term.

To prove Lemma 5.2 , it is enough to prove that there exists a constant $C$ such that for all $t \in[0, T]$,

$$
\|H\|_{L^{\infty}([0, T])}+\|E\|_{L^{\infty}([0, T])} \leq C\left\|X_{1}-X_{2}\right\|_{L^{\infty}([0, T])} .
$$

Indeed, we have with the Hölder inequality

$$
\left\|F_{1}-F_{2}\right\|_{L^{\infty}([0, T])} \leq J T^{\frac{1}{q^{\prime}}}\|\alpha\|_{L^{q}}\left(\|H\|_{L^{\infty}([0, T] \times[0, T])}+\|E\|_{L^{\infty}([0, T] \times[0, T])}\right)
$$


hence

$$
\left\|F_{1}-F_{2}\right\|_{L^{\infty}([0, T])} \leq C T^{\frac{1}{q^{\prime}}}\left\|X_{1}-X_{2}\right\|_{L^{\infty}([0, T])} .
$$

By taking $T$ small enough, we deduce estimate (18). This is where we use $q>1$.

Study of $H$. We have

$$
\begin{gathered}
H=H_{1}+H_{2}+H_{3} \text { where } \\
H_{1}=\int_{0}^{t}\left(N_{1}-N_{2}\right)(t-s) p\left(s, X_{1}(t)\right) e^{-\int_{0}^{s} p\left(s^{\prime}, X_{1}\left(s^{\prime}+t-s\right)\right) d s^{\prime}} d s \\
H_{2}=\int_{0}^{t} N_{2}(t-s)\left(p\left(s, X_{1}(t)\right)-p\left(s, X_{2}(t)\right)\right) e^{-\int_{0}^{s} p\left(s^{\prime}, X_{1}\left(s^{\prime}+t-s\right)\right) d s^{\prime}} d s \\
H_{3}=\int_{0}^{t} N_{2}(t-s) p\left(s, X_{2}(t)\right)\left(e^{-\int_{0}^{s} p\left(s^{\prime}, X_{1}\left(s^{\prime}+t-s\right)\right) d s^{\prime}}-e^{-\int_{0}^{s} p\left(s^{\prime}, X_{2}\left(s^{\prime}+t-s\right)\right) d s^{\prime}}\right) d s .
\end{gathered}
$$

To deal with $H_{1}$, we have to control more precisely $N_{1}-N_{2}$. We have

$$
N_{1}-N_{2}(t)=F_{1}+F_{2}(t)
$$

where

$$
\begin{gathered}
F_{1}(t)=\int_{0}^{+\infty} p\left(s, X_{1}\right)(t)\left(n_{1}(s, t)-n_{2}(s, t)\right) d s \\
F_{2}(t)=\int_{0}^{+\infty} n_{2}(s, t)\left(p\left(s, X_{2}(t)\right)-p\left(s, X_{1}(t)\right)\right) d s .
\end{gathered}
$$

To control the term $F_{2}$, we use the Hölder inequalities, which tells us that

$$
\left|F_{2}(t)\right| \leq\left\|n_{2}(t)\right\|_{L^{\infty}} \mathcal{L}\left\{s \in \mathbb{R}^{+} \text {such that } p\left(s, X_{2}(t)\right)-p\left(s, X_{1}(t)\right) \neq 0\right\}
$$

where $\mathcal{L}$ denotes the Lebesgue measure. But we have

$$
\begin{aligned}
\mathcal{L}\left\{s \in \mathbb{R}^{+} \text {such that } p\left(s, X_{2}(t)\right)-p\left(s, X_{1}(t)\right) \neq 0\right\} & \leq\left|s^{*}\left(X_{2}(t)\right)-s^{*}\left(X_{1}(t)\right)\right| \\
& \leq\left\|s^{* \prime}\right\|_{L^{\infty}}\left|X_{1}(t)-X_{2}(t)\right| .
\end{aligned}
$$

Therefore we have obtained that there exists a constant $C$ such that for all $t \in \mathbb{R}^{+}$

$$
\left|F_{2}(t)\right| \leq C\left|X_{1}(t)-X_{2}(t)\right| \text {. }
$$

To control the term $F_{1}$, we study $\widetilde{n}:=n_{1}-n_{2}$. Using computations made in Section 4 , we deduce that there exists $\mu>0$ such that

$$
f^{\prime}(t) \leq-\mu f(t)+C\left|X_{1}(t)-X_{2}(t)\right|
$$

where $f(t):=\int_{0}^{+\infty} \psi|\widetilde{n}| d s$. As $n_{1}(s, 0)=n_{2}(s, 0)$, we deduce that $f(0)=0$ and using equation $(24)$, we deduce that for all $t \geq 0$,

$$
f(t) \leq 2 \frac{C}{\mu}\left|X_{1}(t)-X_{2}(t)\right|
$$

By consequence, there exists a constant $C$ such that

$$
F_{1}(t) \leq C\left|X_{1}(t)-X_{2}(t)\right|,
$$


which proves that there exists a constant $C$ such that for all $T>0$,

$$
\left\|H_{1}\right\|_{L^{\infty}([0, T])} \leq C\left\|X_{1}-X_{2}\right\|_{L^{\infty}([0, T])} .
$$

Concerning the term $\mathrm{H}_{2}$, we obtain with the Hölder inequality

$$
H_{2}(t) \leq\left\|N_{2}\right\|_{L^{\infty}\left(\mathbb{R}^{+}\right)} \mathcal{L}\left\{s \in \mathbb{R}^{+} \text {such that } p\left(s, X_{2}(t)\right)-p\left(s, X_{1}(t)\right) \neq 0\right\} .
$$

Hence there exists a constant $C$ such that for all $T>0$, for all $t \in[0, T]$

$$
H_{2}(t) \leq C\left\|X_{1}-X_{2}\right\|_{L^{\infty}([0, T])}
$$

To deal with the term $H_{3}$, we use the fact that there exists a constant $C$ such that

$$
\left|e^{-\int_{0}^{s} p\left(s^{\prime}, X_{1}\left(s^{\prime}+t-s\right)\right) d s^{\prime}}-e^{-\int_{0}^{s} p\left(s^{\prime}, X_{2}\left(s^{\prime}+t-s\right)\right) d s^{\prime}}\right| \leq C \int_{0}^{s}\left|p\left(s^{\prime}, X_{1}\left(s^{\prime}+t-s\right)\right)-p\left(s^{\prime}, X_{2}\left(s^{\prime}+t-s\right)\right)\right| d s^{\prime} .
$$

We deduce that there exists a constant $C$ such that

$$
\left|H_{3}(t)\right| \leq C \int_{0}^{t}\left(\int_{0}^{t}\left|p\left(s^{\prime}, X_{1}\left(s^{\prime}+t-s\right)\right)-p\left(s^{\prime}, X_{2}\left(s^{\prime}+t-s\right)\right)\right| d s\right) d s^{\prime} .
$$

With the change of variable $v=s^{\prime}+t-s$, we deduce that

$$
\left|H_{3}(t)\right| \leq C \int_{0}^{t}\left(\int_{s^{\prime}}^{t}\left|p\left(s^{\prime}, X_{1}(v)\right)-p\left(s^{\prime}, X_{2}(v)\right)\right| d v\right) d s^{\prime}
$$

and hence

$$
\left|H_{3}(t)\right| \leq C \int_{0}^{t}\left(\int_{0}^{t}\left|p\left(s^{\prime}, X_{1}(v)\right)-p\left(s^{\prime}, X_{2}(v)\right)\right| d v\right) d s^{\prime} .
$$

Using the fact that there exists a constant $C$ such that for all $v \in[0, t]$

$$
\mathcal{L}\left\{s \in \mathbb{R}^{+} \text {such that } p\left(s, X_{2}(v)\right)-p\left(s, X_{1}(v)\right) \neq 0\right\} \leq\left\|s^{* \prime}\right\|_{L^{\infty}}\left|X_{1}(v)-X_{2}(v)\right|,
$$

we deduce that there exists a constant $C$ such that

$$
\left|H_{3}(t)\right| \leq C\left\|X_{1}(t)-X_{2}(t)\right\|_{L^{\infty}([0, T])} .
$$

Study of $E$. We have

$$
E(t)=\int_{t}^{+\infty} n^{0}(s-t)\left(p\left(s, X_{1}(t)\right) e^{-\int_{0}^{t} p\left(t^{\prime}+s-t, X_{1}\left(t^{\prime}\right)\right) d t^{\prime}}-p\left(s, X_{2}(t)\right) e^{-\int_{0}^{t} p\left(t^{\prime}+s-t, X_{2}\left(t^{\prime}\right)\right) d t^{\prime}}\right) d s .
$$

As $\left\|n^{0}\right\|_{L^{\infty}} \leq 1$, it is enough to prove that there exists a constant $C$ such that for all $t \in[0, T]$

$$
\begin{aligned}
G & :=\int_{t}^{+\infty}\left|p\left(s, X_{1}(t)\right) e^{-\int_{0}^{t} p\left(t^{\prime}+s-t, X_{1}\left(t^{\prime}\right)\right) d t^{\prime}}-p\left(s, X_{2}(t)\right) e^{-\int_{0}^{t} p\left(t^{\prime}+s-t, X_{2}\left(t^{\prime}\right)\right) d t^{\prime}}\right| d s \\
& \leq C\left\|X_{1}-X_{2}\right\|_{L^{\infty}([0, T])} .
\end{aligned}
$$

We have

$$
G \leq G_{1}+G_{2}
$$


where

$$
\begin{gathered}
G_{1}=\int_{t}^{+\infty}\left|p\left(s, X_{1}(t)\right)-p\left(s, X_{2}(t)\right)\right| e^{-\int_{0}^{t} p\left(t^{\prime}+s-t, X_{1}\left(t^{\prime}\right)\right) d t^{\prime}} d s, \\
G_{2}=\int_{t}^{+\infty} p\left(s, X_{2}(t)\right)\left|e^{-\int_{0}^{t} p\left(t^{\prime}+s-t, X_{2}\left(t^{\prime}\right)\right) d t^{\prime}}-e^{-\int_{0}^{t} p\left(t^{\prime}+s-t, X_{1}\left(t^{\prime}\right)\right) d t^{\prime}}\right| d s .
\end{gathered}
$$

Using estimate (22), we deduce that there exists a constant $C$ such that

$$
G_{1} \leq C\left\|X_{1}-X_{2}\right\|_{L^{\infty}([0, T])}
$$

We have

$$
G_{2} \leq \int_{t}^{+\infty} \int_{0}^{t}\left|p\left(t^{\prime}+s-t, X_{2}\left(t^{\prime}\right)\right)-p\left(t^{\prime}+s-t, X_{1}\left(t^{\prime}\right)\right)\right| d t^{\prime} d s .
$$

By the change of variables $v=t^{\prime}+s-t$, we deduce that

$$
G_{2} \leq C \int_{0}^{+\infty} \int_{0}^{t}\left|p\left(v, X_{2}\left(t^{\prime}\right)\right)-p\left(v, X_{1}\left(t^{\prime}\right)\right)\right| d v d t^{\prime}
$$

which proves that there exists a constant $C$ such that

$$
G_{2} \leq C\left\|X_{1}-X_{2}\right\|_{L^{\infty}([0, T])}
$$

using estimate (22). All these estimates together complete the proof Lemma 5.2.

It remains to prove estimate (17) of Theorem 5.1. Firstly, we have

$$
N(t)=\int_{0}^{+\infty} p(s, X(t)) n(s, t) d s \leq \int_{0}^{+\infty} n(s, t) d s=1 .
$$

Secondly, for $X(t)$, we write

$$
X(t)=J \int_{0}^{+\infty} \alpha(u) N(t-u) d u \leq J\|N\|_{L^{\infty}} \int_{0}^{+\infty} \alpha(u) d u \leq J .
$$

On the other hand, we have

$$
N(t) \geq \int_{s_{+}^{*}}^{+\infty} n(s, t) d s
$$

because $p(s, \cdot) \equiv 1$ for all $s \in\left[s_{+}^{*},+\infty\right)$. But,

$$
\int_{0}^{s_{+}^{*}} n(s, t) d s \leq s_{+}^{*}\|n\|_{L^{\infty}} \leq s_{+}^{*} \max \left(\left\|n^{0}\right\|_{L^{\infty}},\left\|n^{0}\right\|_{L^{1}}\right) \leq s_{+}^{*} .
$$

As for all $t \geq 0$, we know that $\int_{0}^{+\infty} n(s, t) d s=1$, we deduce that

$$
\int_{s_{+}^{*}}^{+\infty} n(s, t) d s \geq 1-s_{+}^{*} .
$$

We deduce that

$$
X(t) \geq J\left(1-s_{+}^{*}\right)
$$

which completes the proof of Theorem 5.1. 


\section{Nonlinear stability of the steady state for $J \approx 0$ and $J \approx+\infty$.}

In this section we prove the nonlinear asymptotic stability of the steady state on the one hand for $J$ small enough, and on the other hand for $J \approx+\infty$. For $J \approx+\infty$ our analysis is based on the decay property $(10)$ of $s^{*}(x)$ for $\widetilde{D}$ small enough.

Our approach uses the spectral gap property arising from Lemma 4.2. Given a steady state $X_{J}^{*}$, we can consider the value $s^{*}\left(X_{J}^{*}\right) \in\left(s_{-}^{*}, s_{+}^{*}\right)$, and the corresponding function $\psi_{J} \geq B>0$ as in Lemma 4.2. We recall that the constants $\nu>0, \mu>0$ and $B>0$ can be chosen uniformly positive, bounded, and independent of $J$. For this reason we use the notation $\psi$ in place of $\psi_{J}$ in this section.

The main theorem of this section is the following

Theorem 6.1 Assume (2), (8) with $s_{+}^{*}<1$ and

$$
\int_{0}^{\infty} \psi(s)\left|n^{0}(s)-A_{J}(s)\right| d s<+\infty
$$

where $A_{J}$ is given by equation (12) and consider the solution $n$ to (1), (3).

Then, for $J$ small enough, the following estimate holds

$$
\lim _{t \rightarrow+\infty} \int_{0}^{\infty} \psi(s)\left|n(s, t)-A_{J}(s)\right| d s=0
$$

For J large enough, and assuming that estimate (10) holds with $\widetilde{D}$ small enough, the following estimate holds

$$
\lim _{t \rightarrow+\infty} \int_{0}^{\infty} \psi(s)\left|n(s, t)-A_{J}(s)\right| d s=0 .
$$

We recall, from section 3 that these are regimes where the steady states are unique. We first treat the case with no delay which is simpler and allows us to give the idea of the proof. Then, we consider time delay.

\subsection{The case with no delay}

Theorem 6.2 Assume (2), (8) with $s_{+}^{*}<1$ and that there is no delay, i.e., $X(t)=J N(t)$. Then, there is a constant $\beta>0$ such that for $J$ small enough, the following estimate holds

$$
\int_{0}^{\infty} \psi(s)\left|n(s, t)-A_{J}(s)\right| d s \leq e^{-\beta t} \int_{0}^{\infty} \psi(s)\left|n^{0}(s)-A_{J}(s)\right| d s
$$

where $A_{J}$ is given by equation (12).

Proof of Theorem 6.2. The function $\widetilde{n}=n-A_{J}$ satisfies the equation

$$
\partial_{t} \tilde{n}+\partial_{s} \widetilde{n}+p\left(s, X_{J}^{*}\right) \widetilde{n}+\left[p(s, X(t))-p\left(s, X_{J}^{*}\right)\right] n=0 .
$$

We deduce that

$$
\partial_{t}|\widetilde{n}|+\partial_{s}|\widetilde{n}|+p\left(s, X_{J}^{*}\right)|\widetilde{n}| \leq\left|p(s, X(t))-p\left(s, X_{J}^{*}\right)\right| n .
$$


In order to simplify notations, we define $f$ by

$$
f(t):=\int_{0}^{\infty} \psi|\widetilde{n}(s, t)| d s=\int_{0}^{\infty} \psi(s)\left|n(s, t)-A_{J}(s)\right| d s .
$$

Then, according to the calculation in the proof of Theorem 4.1,

$$
\frac{d}{d t} f(t) \leq-\mu f(t)+\int_{0}^{\infty} \mathbb{I}_{s \in\left[s^{*}(X(t)), s^{*}\left(X_{J}^{*}\right)\right]} \psi n(s, t) d s
$$

As the function $s^{*}(\cdot)$ is Lipschitz continuous, and $0 \leq n \leq 1$, if we note $C_{J}=\left\|s^{* \prime}\right\|_{L^{\infty}(\mathcal{C}(J))}$ (where $\mathcal{C}(J)$ is given by $(11)$ ); we deduce that there exists a constant $C$ such that

$$
\int_{0}^{\infty} \mathbb{I}_{s \in\left[s^{*}(X(t)), s^{*}\left(X_{J}^{*}\right)\right]} \psi n(s, t) d s \leq C_{J}\left|X(t)-X_{J}^{*}\right|\|\psi n\|_{L^{\infty}\left(s_{-}^{*}, s_{+}^{*}\right)} \leq C C_{J}\left|X(t)-X_{J}^{*}\right| .
$$

But, we have also

$$
X(t)-X_{J}^{*}=J \int_{0}^{+\infty} p(s, X(t)) \widetilde{n} d s+J \int_{0}^{+\infty}\left[p(s, X(t))-p\left(s, X_{J}^{*}\right)\right] A_{J}(s) d s .
$$

We deduce that

$$
\left|X(t)-X_{J}^{*}\right| \leq \frac{J}{B} f(t)+C_{J} J\left|X(t)-X_{J}^{*}\right|\left\|A_{J}\right\|_{L^{\infty}}
$$

and hence, since $\left\|A_{J}\right\|_{L^{\infty}}=A_{J}(0)<1$, we have as long as $C_{J} J<1$,

$$
\left|X(t)-X_{J}^{*}\right| \leq \frac{J}{B\left(1-C_{J} J\right)} f(t)
$$

Back to (25), we obtain

$$
\frac{d}{d t} f(t) \leq-\mu f(t)+C \frac{C_{J} J}{B\left(1-C_{J} J\right)} f(t)
$$

which proves Theorem 6.2 under the condition $C_{\frac{C_{J} J}{B\left(1-C_{J} J\right)}}<\mu$; that is to say that $C_{J} J$ is small enough. This is the case as soon as $J$ is small enough because $C_{J} \leq\left\|s^{* \prime}\right\|_{L^{\infty}\left(\mathbb{R}^{+}\right)}$remains bounded. This is also the case when $J$ is large enough and if condition (10) holds with $\widetilde{D}$ small enough.

\subsection{Case with delay}

Proof of Theorem 6.1. In this setting, it is a priori not true that the solution converge exponentially to the steady state given by equation (12). We are going to prove that the solution tends to the steady state when $t \rightarrow+\infty$ without informations of the rate of convergence. This is due to the fact that here, we add a delay pondered by the function $\alpha$ in the formula of $X$ with respect to the preceding case, and the way that the solution tends to the steady state at infinity seems depends strongly of the function $\alpha$. To prove Theorem 6.1, the idea is to use an iteration proceed.

Using formula (25) in the proof of Theorem 6.2, we already know that

$$
\frac{d}{d t} f(t) \leq-\mu f(t)+C C_{J}\left|X(t)-X_{J}^{*}\right|
$$


The problem here is to control the term $\left|X(t)-X_{J}^{*}\right|$ which do not trivially tends to 0 when $t$ tends to $+\infty$ because the formula of $X(t)$ takes in consideration all the value of $f$ between 0 and $t$ contrarily to the case with no delay. But we have the following control on $X$ and $X_{J}^{*}$, see (17),

$$
X(t) \in \mathcal{C}(J) \quad \text { and } \quad X_{J}^{*} \in \mathcal{C}(J)
$$

and therefore

$$
\frac{d}{d t} f(t) \leq-\mu f(t)+C\left(C_{J} J\right)
$$

Hence, if $J$ is small enough or if $J$ is big enough and if condition (10) holds with $\widetilde{D}$ small enough, then $C_{J} J$ is small. We can deduce that $f$ is also not to big if $t$ is big enough. More precisely

$$
f(t) \leq \max \left(f(0), \frac{C\left(C_{J} J\right)}{\mu}\right), \quad f(t) \leq 2 \frac{C\left(C_{J} J\right)}{\mu} \text { for } t \geq T_{0} \text { large enough. }
$$

Now we can estimate more accurately the term

$$
\begin{aligned}
X(t)-X_{J}^{*} & =J \int_{0}^{+\infty} \alpha(u) \int_{0}^{+\infty} p(s, X(t-u)) \widetilde{n}(s, t-u) d s d u \\
& +J \int_{0}^{+\infty} \alpha(u) \int_{0}^{+\infty}\left[p(s, X(t-u))-p\left(s, X_{J}^{*}\right)\right] A_{J}(s) d s d u .
\end{aligned}
$$

We claim that in fact if $t$ is large enough then a constant $C$ exists such that

$$
\left|X(t)-X_{J}^{*}\right| \leq C \frac{C_{J} J^{2}}{\mu}
$$

To prove it, we set

$$
\begin{gathered}
I_{1}(t):=\int_{0}^{+\infty} \alpha(u) \int_{u=0}^{t} p(s, X(t-u))|\widetilde{n}(s, t-u)| d s d u, \\
I_{2}(t):=\int_{u=0}^{t} \alpha(u) \int_{0}^{\infty}\left|p(s, X(t-u))-p\left(s, X^{*}\right)\right| A_{J}(s) d s d u .
\end{gathered}
$$

Concerning, the second term $I_{2}(t)$, using the fact that at $t$ and $u$ fixed, the set of $s \in \mathbb{R}^{+}$such that $\left|p(s, X(t-u))-p\left(s, X^{*}\right)\right| \neq 0$ is less than $\left|s^{*}(X(t-u))-s^{*}\left(X_{J}^{*}\right)\right|$; we deduce via (26) (which holds for all $t)$ that for all $(t, u) \in \mathbb{R}^{+} \times[0, t]$

$$
\left|s^{*}(X(t-u))-s^{*}\left(X_{J}^{*}\right)\right| \leq\left(C_{J} J\right) .
$$

Hence, we deduce that

$$
0 \leq I_{2} \leq 2\left(C_{J} J\right)\left\|A_{J}\right\|_{L^{\infty}} \leq 2\left(C_{J} J\right) .
$$

Remark 6.3 Let us mention that here, (26) holds for all $t \in \mathbb{R}^{+}$and hence we do not see the problem of the delay. For the second iteration, we control estimate (29) only if $t$ is big enough so we can not apply exactly the same strategy and we have to deal with the delay. The question of the delay is treated in the next estimate of $I_{2}$ where the problem of the delay appear in the first iteration. 
Concerning, the first term $I_{1}$, we have

$$
0 \leq I_{1}(t) \leq \frac{1}{B} \int_{u=0}^{t} \alpha(u) \int_{0}^{\infty} \psi(s)|\widetilde{n}(s, t-u)| d s d u .
$$

leq Here, we must be careful of the delay because here at $t$ fixed, $f$ is integrated over all the points $t^{\prime}=t-u$ with $u \in[0, t]$ that is to say that all the values of $f$ between 0 and $t$ are taken in consideration. But we only know that $f\left(t^{\prime}\right)$ is small if $t^{\prime}$ is big enough. This is in fact not a problem because the function $\alpha \in L^{1}$. Indeed, when $t$ is big, $t-u$ is small (and hence bad) only when $u$ is near to $t$. But $\alpha$ being integrable, we have

$$
\lim _{A \rightarrow+\infty} \int_{A}^{+\infty} \alpha(u) d u=0
$$

More precisely, if $t$ is large enough we have

$$
\alpha * f \leq \frac{2\left(C_{J} J\right)}{\mu}
$$

because $\alpha \in L^{1}$ and $\|f\|_{L^{\infty}} \leq C$. Indeed, we split the integral

$$
\int_{0}^{+\infty} \alpha(u) f(t-u) d u
$$

in two parts. As $\alpha \in L^{1}$, we deduce that there exists a $T_{1}>0$ such that

$$
\int_{T_{1}}^{+\infty} \alpha(u) d u \leq \frac{C_{J} J}{\max \left(1,\|f\|_{L^{\infty}\left(\left[0, T_{0}\right]\right)}\right)},
$$

Let us mention, using (27) that the denominator is controlled because

$$
\|f\|_{L^{\infty}\left(\mathbb{R}^{+}\right)} \leq \max \left(f(0), 2\left(\frac{C C_{J}}{\mu}\right)\right) .
$$

Now, we choose $t$ big enough such that $t-T_{1} \geq T_{0}$ and we have

$$
\int_{0}^{T_{1}} \alpha(u) f(t-u) d u \leq\|\alpha\|_{L^{1}}\|f\|_{L^{\infty}\left(\left[T_{0},+\infty\right]\right)} \leq \frac{C\left(C_{J} J\right)}{\mu} .
$$

Back to our estimate, we obtain

$$
\frac{d}{d t} f(t) \leq-\mu f(t)+C \frac{\left(C_{J} J\right)^{2}}{\mu} .
$$

and hence for $t$ large enough

$$
f(t) \leq C \frac{\left(C_{J} J\right)^{2}}{\mu^{2}}
$$

By taking $J$ small enough or big enough assuming estimate (10) with $\widetilde{D}$ small enough, we obtain that

$$
\frac{C\left(C_{J} J\right)}{\mu}<1
$$

and hence by iteration, we deduce Theorem 6.1 .

Remark 6.4 Starting the second iteration, when we deal with the term $I_{1}$, we also have to split the integral of $\alpha$ in two subintegrals as done for the study of $I_{2}$ for the first iteration because estimate (29) holds only if $t$ is large enough. 

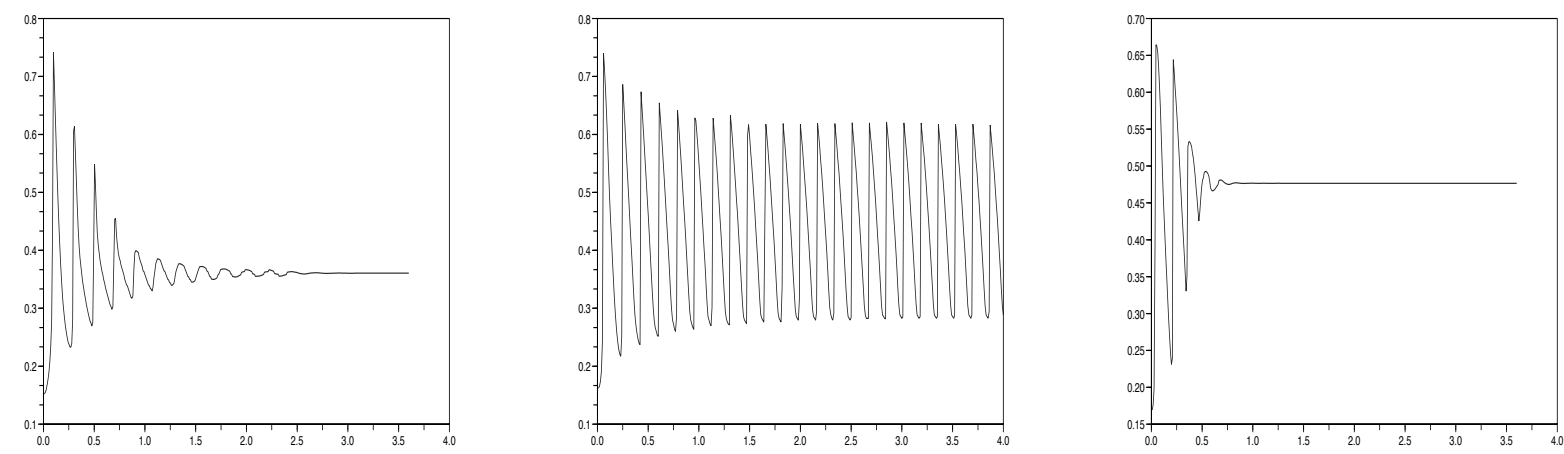

Figure 1: Total neural activity $N(t)$ in the case with no delay. Three different values of the network connectivity have been used, left: $J=2$, center $J=3$, right $J=3.5$.

\section{Periodic solutions and numerical results}

We have seen that the network connectivity $J$ in the neuron system (1) determines both the number of steady states and their stability in the range $J$ small or large. We now study, by means of direct numerical simulations, if other solutions may exist in the middle range of $J$. In particular, despite the dissipative nature of each individual neuron leading to relaxation towards an asynchronous state $(J=0)$, is it possible that the network connectivity leads to natural periodic oscillations as observed experimentally and described by other network models ([1] and the references therein). Existence of periodic solutions for age structured equations has been obtained through Hopf bifurcation, see [8], but the mechanism here is different because it seems to stem from the appearance of several steady states according to section 3 .

We have chosen the framework of the particular firing rate given by formula (8) with

$$
s^{*}(x)=2-\frac{x^{4}}{1+x^{4}},
$$

and we study either the case with no delay or a Poisson law for the pulse propagation delay $\alpha$

$$
\alpha(u)=e^{-a u} .
$$

The numerical solutions are depicted in Figure 1 . They put in evidence that an intermediate regime exists for appropriate values of $J$ where periodic solutions occur.

The effect of delay is generically to stabilize the dynamics and the periodic solutions do not seem to exist for $J=3$ for instance. The numerical solutions for $J=2.5$ are depicted in Figure 2 and show that periodic solutions persist for large enough values of $a$ in (30).

\section{References}

[1] Brunel, N. Dynamics of sparsely connected networks of excitatory and inhibitory spiking networks. J. Comp. Neurosci. 8 (2000) 183-208.

[2] Diekmann O. and Gyllenberg M. The second half- with a quarter of a century delay. Math. Model. Nat. Phenom. Vol.3 No. 7 (2008), 36-48. 

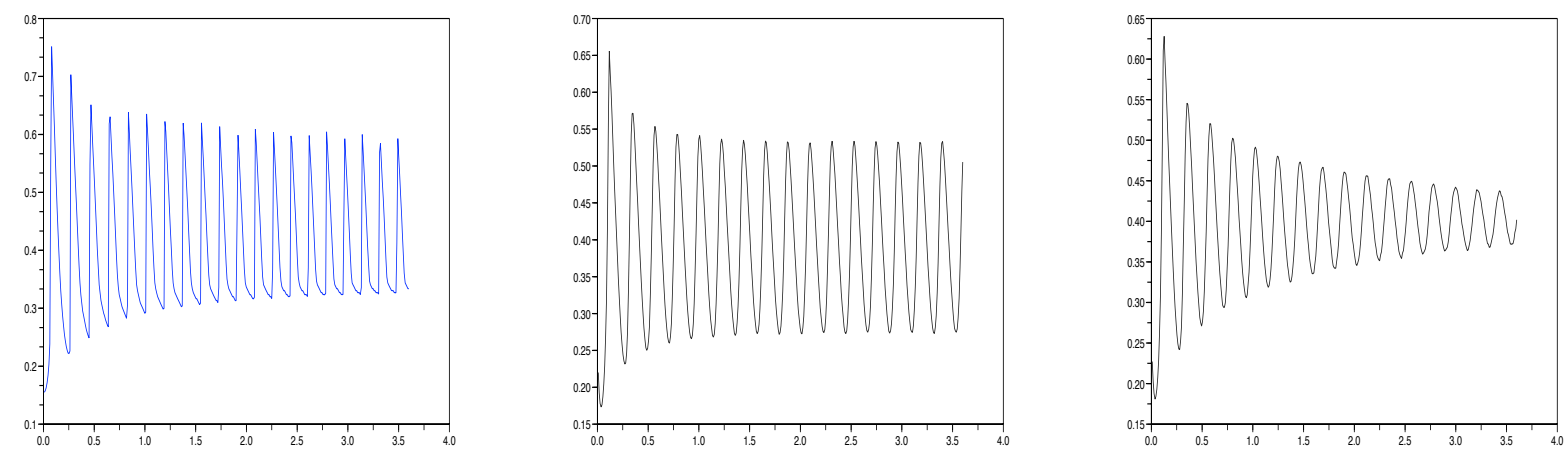

Figure 2: Total neural activity $N(t)$ in the case with delay. We used the network connectivity $J=2.5$. Left: no delay, center $a=5$, right $a=4$.

[3] Diekmann O., Getto, P. and Gyllenberg M. Stability and bifurcation analysis of Volterra functional equations in the light of suns and stars. Siam J. Math. Anal. Vol. 39, No. 4 (2007) 1023-1069.

[4] Faugeras, O., Grimbert, F. and Slotine J.-J. Stability and synchronization in neural fields. In Sixteenth Annual Computational Neuroscience Meeting (CNS), Toronto, Jul. 2007,

[5] Feller, W. An introduction to probability theory and applications. Wiley, New-York (1966).

[6] Gyllenberg, M., Webb, G.F. A nonlinear structured population model of tumor growth with quiescence. J. Math. Biol. 28:671-694 (1990).

[7] Hien L. V., Loan T. T. and Tuan D. A. Periodic solutions and exponential stability for shunting inhibitory cellular neural networks with continuously distributed delays. Electronic journal of differential equations, Vol. 2008 (2008), No. 7, 1-10.

[8] Magal, P. and Ruan S. Center manifold for semilinear equations with non-dense domain and applications on Hopf bifurcation in age structured models. Memoirs Amer. Math. Soc. To appear.

[9] Michel, P., Mischler, S., Perthame, B. General relative entropy inequality: an illustration on growth models, J. Math. Pures Appl. 84(9):1235-1260 (2005).

[10] Murray, J. D., Mathematical biology, Vol. 1 and 2, Second edition. Springer (2002).

[11] Perthame, B., Transport equations in biology. Series 'Frontiers in Mathematics', Birkhauser (2007)

[12] Pham, J., Pakdaman, K., Champagnat, J. and Vivert, J.-F. Activity in sparsely connected excitatory neural networks: effect of connectivity. Neural Networks 11 (1998) 415-434.

[13] Pham, J., Pakdaman, K. and Vivert, J.-F. Noise-induced coherent oscillations in randomly connected neural networks. Phys. Rev. E 58(3) (1998) 415-434.

[14] van Ooyen A., van Pelt J., Corner M. A., da Silva F. H. The emergence of long-lasting transients of activity in simple neural networks. Biol Cybern. 1992;67(3):269-77. Erratum in: Biol Cybern 1992;68(1):93. 